\title{
INFLUENCIA DEL EXOESQUELETO DE MIEMBRO INFERIOR EN SEÑALES EEG
}

\author{
M. Rodríguez-Ugarte*1, E. Iáñez ${ }^{1}$, M. Ortiz ${ }^{1}$, J. M. Cano ${ }^{2}$, J. A. Flores ${ }^{3}, J$. M. Azorín ${ }^{1}$ \\ ${ }^{1}$ Brain-machine Interface Systems Lab. Universidad Miguel Hernández de Elche. \\ Avda. de la Universidad S/N, 03202 Elche, España \\ \{maria.rogriguezu, eianez, mortiz, jm.azorin\}@umh.es \\ 2 Departamento de Ingeniería de Sistemas y Automática, Universidad Politécnica de Cartagena. \\ Campus Muralla del Mar S/N. 30202 Cartagena, España. \\ josem.cano@upct.es \\ 3 Departamento de Ingeniería, Universidad Miguel Hernández. \\ Crta. Beniel, Km. 3,2 - Orihuela, 03312, España \\ ja.flores@umh.es
}

\section{Resumen}

El accidente cerebrovascular (ACV) es una de las principales causas en el mundo que provoca discapacidad. Por ello, la comunidad cientifica está investigando la manera de ayudar a los afectados de $A C V$. Este trabajo utiliza las interfaces cerebro-máquina (BMI) y un exoesqueleto de miembro inferior para mejorar el proceso de rehabilitación de dichas personas. De esta manera, el futuro paciente, estará más involucrado en su terapia de rehabilitación. El objetivo de este trabajo es estudiar si el ruido que general el movimiento del exoesqueleto afecta al EEG de las señales adquiridas del usuario y por ende verse influenciado en el desempeño de la BMI. Se ha realizado un estudio del espectro de la potencia de las señales utilizando la Transformada rápida de Fourier. Los resultados indican que dicho movimiento, no genera una diferencia significativa en la potencia obtenida de los electrodos seleccionados. Sin embargo, un grupo de electrodos en el área occipital sí que presentaba diferencias significativas. En el futuro, se tendrá en cuenta dicha información cuando se utilice esta tecnología con personas que han sufrido un $A C V$.

Palabras clave: Exoesqueleto, EEG, interfaz cerebro-máquina, ruido

\section{INTRODUCCIÓN}

El accidente cerebrovascular (ACV) es una de las principales causas en el mundo que provoca discapacidad [15]. Aproximadamente, entre el 30-40\% de los supervivientes de ACV tienen limitaciones en su capacidad para caminar, afectando a su vida cotidiana [9], [16]. Por ello, es necesario avanzar en la investigación de la rehabilitación de la marcha para aquellas personas que han sufrido un ACV.

En los últimos años, la comunidad científica está desarrollando diferentes métodos para mejorar la calidad de vida de personas que han sufrido un ACV. Por ejemplo, se están usando exoesqueletos para dar soporte a la extremidad afectada [6], [14]. Entre ellos, los más comunes son los exoesqueletos de brazos y piernas. Por otra parte, se están investigando interfaces cerebro-máquina (Brain-machine interface en inglés, BMI) [20], [18]. Las BMI traducen las señales cerebrales, comúnmente conocidas como señales electroencefalográficas (EEG) en comandos para gobernar un dispositio externo. Así pues, si unimos el exoesqueleto previamente mencionado a las BMI, supondría la creación de un camino alternativo para controlar el movimiento de las piernas. La combinación de esta tecnología, permite a los pacientes estar más involucrados en su terapia de rehabilitación.

Existen dos paradigmas principales de BMI basados en EEG: ejecución motora (motor execution en inglés, ME) e imaginación motora (motor imagery en inglés, MI) [8]. La diferencia entre ambas es que mientras $\mathrm{ME}$ se requiere realizar una tarea de movimiento, durante MI se imaginación la realización de dicho movimiento sin llegar a ejecutarlo. Sin embargo, se ha investigado que ambas estrategias activan caminos neuronales comunes; entre ellos la corteza primaria motora (M1), la corteza somatosensorial primaria (S1), la corteza premotora (premotor cortez en inglés, PM) y el área motora suplementaria (supplementary motor area en inglés, SMA) [1]. Además, durante MI existe atenuación en las bandas de frecuencia $\theta(5-7 \mathrm{~Hz}), \alpha(8-15 \mathrm{~Hz})$ and $\beta \quad(16-30 \mathrm{~Hz}) \quad[2] \quad[20]$. Esta inhibición de potencia es causada por la desincronización de las ondas cerebrales, comúnmente conocidas como desincronización de eventos relacionados 
(event-related desynchronization en inglés, ERD). Por lo tanto, MI es una buena opción para personas que experimentan discapacidad motora para activar un exoesqueleto.

El objetivo de este trabajo es estudiar si el ruido que general el movimiento del exoesqueleto afecta al EEG de las señales adquiridas del usuario y por ende verse influenciado en el desempeño de la BMI. La BMI está basado en señales EEG para activar el exoesqueleto de miembro inferior y clasifica dos estados de imaginación: relajación e imaginación de la marcha.

\section{MATERIALES Y MÉTODOS}

En este estudio, un voluntario diestro sin ningún tipo de desorden neuronal participó en este experimento. El usuario fue informado del estudio que se estaba llevando a cabo y dió su consentimiento informado para realizar dichas pruebas. El estudio fue aprobado por el Comité de Ética de la Universidad Miguel Hernández de Elche (España).

\section{$2.1 \quad$ PROTOCOLO EXPERIMENTAL}

El participante tenía que realizar tareas de relajación e imaginación de marcha mientras se estaban grabando sus señales EEG. El mismo protocolo tuvo que realizarlo en dos escenarios diferentes: sólo con el gorro de EEG (E1) y con el gorro de EEG y el exoesqueleto (E2). Ambos escenarios son explicados con más detalle en el siguiente apartado.

El protocolo para ambos escenario era el siguiente: dos periodos de relajación ( $\mathrm{R} 1$ y $\quad \mathrm{R} 2)$ de 7 segundos cada uno, separados por un periodo de imaginación de marcha (GMI) de 17 segundos. Primero, se le indicaba al usuario el inicio de la prueba con R1. Posteriormente, un pitido definía el comienzo de GMI. El fin del periodo de GMI y comienzo de R2 era indicado mediante dos pitidos. Durante la relajación, el usuario tenía que concentrarse en la respiración mientras que durante GMI, tenía que imaginar que estaba andando.

El usuario realizó 20 repeticiones por cada escenario del protocolo experimental.

\subsection{ESCENARIOS}

1. E1: El usuario estaba de pie enfrente de una pared blanca con el gorro de EEG grabando sus señales mientras realizaba el protocolo experimental. El participante no llevaba puesto el exoesqueleto de miembro inferior.
2. E2: El usuario realizó el protocolo experimental con el gorro de EEG y el exoesqueleto de miembro inferior. Durante los periodos de GMI, además de estar imaginando que andaba, el exoesqueleto andaba por sí mismo para dar una sensación más realista al usuario. La Figura 1 muestra el protocolo experimental de este escenario.

\subsection{ANÁLISIS DE LOS DATOS}

Para los dos escenarios se utilizó el equipo StarStim R32 de Neuroelectrics (c). Se registraron 30 canales de EEG situados como el Sistema Internacional 10-10 a una frecuencia de muestreo de $500 \mathrm{~Hz}$.

Para estudiar si el movimiento del exoesqueleto produce alguna alteración en el EEG del usuario, se ha realizado un estudio de la potencia espectral. Primero, los datos fueron separados en Relajación e Imaginación. Posteriormente, los primeros dos segundos de cada tarea fueron descartados para eliminar la influencia de la señal auditiva en el EEG. A continuación, las señales se procesaron en epochs de 1 segundo cada 0.2 segundos. A cada epoch se le aplicó: un filtro Notch a 50 $\mathrm{Hz}$ para eliminar la influencia de la red eléctrica, un filtro pasa banda entre 0.05 y $45 \mathrm{~Hz}$ para quedarnos con la banda de frecuencia deseada y un filtro Laplaciano como en el estudio de McFarland et. al. [11] para eliminar la influencia de los otros electrodos. Después, se seleccionaron los 9 electrodos de interés, que son aquellos que están situados donde se produce las mayores variaciones cuando se realiza imaginación de una tarea motora: $\mathrm{Cz}, \mathrm{CP} 1, \mathrm{CP} 2, \mathrm{C} 1, \mathrm{C} 2, \mathrm{C} 3, \mathrm{C} 4$, FC1 y FC2 (Figura 2). Para esos electrodos, se realizó la Tranformada de Fourier Rápida (Fast Fourier Transform en inglés, FFT) entre 6 y 30 $\mathrm{Hz}$. Este rango de frecuencias fue seleccionado por ser donde se generan mayores variaciones durante la imaginación del movimiento.

\subsection{EXOESQUELETO}

El exoesqueleto utilizado fue el H2 (Technaid, Madrid, España) diseñado por Bortole et al. [3]. El exoesqueleto $\mathrm{H} 2$ tiene 6 grados de libertad donde la cadera, la rodilla y el tobillo de cada pierna son articulaciones motorizadas. Fue construídos para adultos de peso máximo $100 \mathrm{~kg}$ y altura entre 1,5 y $1,95 \mathrm{~m}$.

\subsection{ANÁLISIS ESTADÍSTICO}

Para comprobar si existen diferencias significativas entre E1 y E2, se ha realizado un t-test. Este análisis estadístico comprueba si 


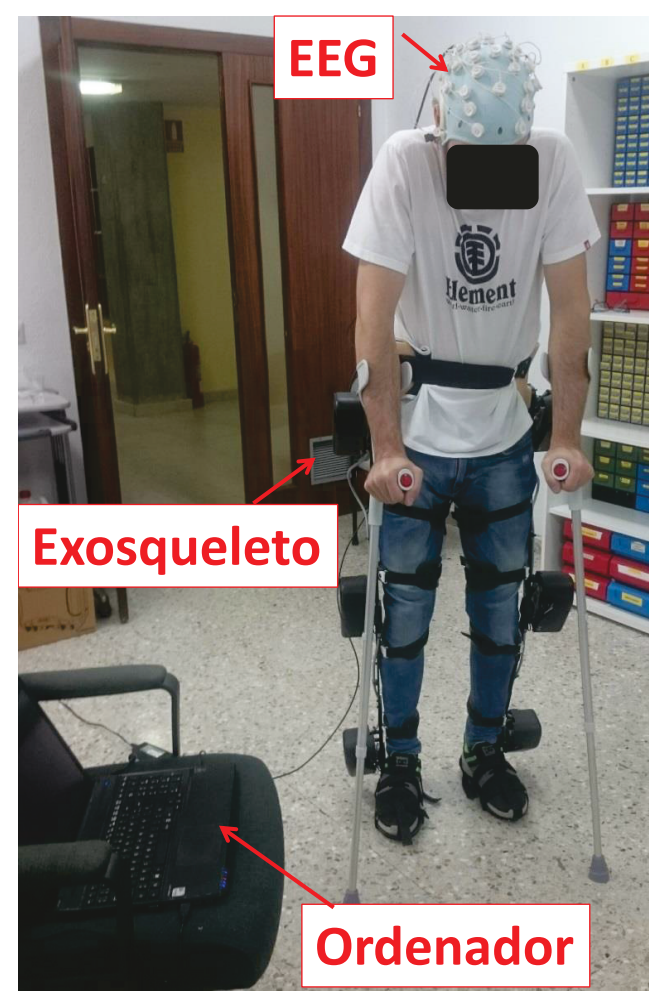

Figura 1: Montaje experimental del escenario 2 (E2). El usuario realiza las tarea de relajación e imaginación mientras se graban sus señales EEG. Durante el periodo de imaginación de la marcha, además, el exoesqueleto se mueve por si mismo.

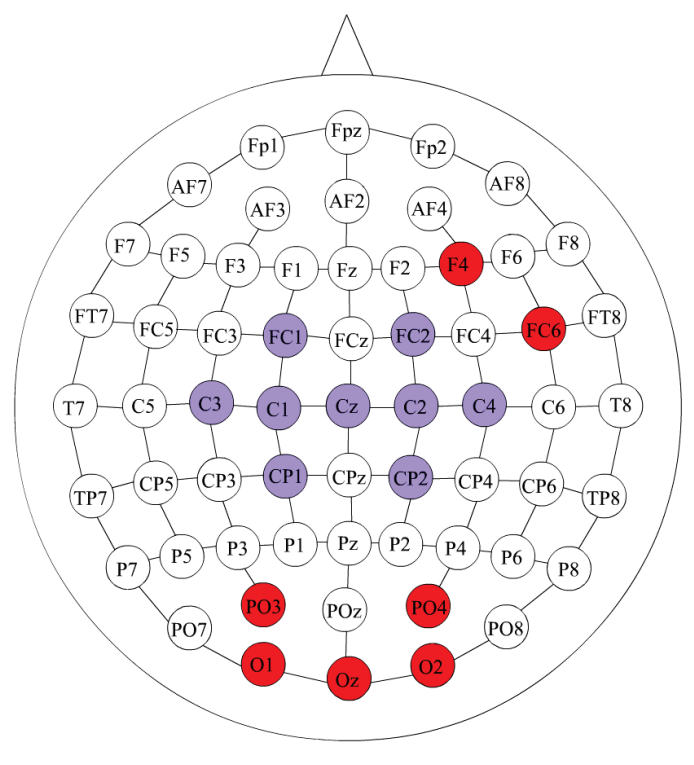

Figura 2: Sistema internacional electrodos 10-10. Coloreados en azul los electrodos finalmente seleccionados.

las medias de ambas señales son iguales o no. Si el p-value $<0.05$, significa que las señales son significativamente diferentes.

\section{RESULTADOS}

Como durante el periodo de relajación, el exoesqueleto no se movía, no producía ningún ruido y por lo tanto las señales de EEG producidas tanto en E1 como en E2 no debería de provocar ninguna alteración. Por ello, se muestra sólo, en la Figura 3, los resultados de la potencia espectral entre 6 y $30 \mathrm{~Hz}$ durante la imaginación de la marcha de cada electrodo. La línea azul se corresponde a E1 y la verde a E2.

Además, la Tabla 1 muestra el valor de significancia (p-value) de cada electrodo del análisis t-test realizado. Asímismo, la Figura 4 muestra la potencia media espectral de tres electrodos ruidosos.

\section{DISCUSIÓN Y CONCLUSIÓN}

Los resultados de la Tabla 1 muestran las significancia de la señales. Con esta información y viendo la posición donde se ecuentran los electrodos ruidosos (Figura 2 y Figura 4), se puede concluir que aquellos situados sobre el lóbulo occipital, presentan mayores significancia entre las señales. Esto puede ser debido a que dicha área del cerebro se encarga de procesar los datos visuales [13]. Por ello, si en algún momento el 
Potencia espectral de los electrodos seleccionados
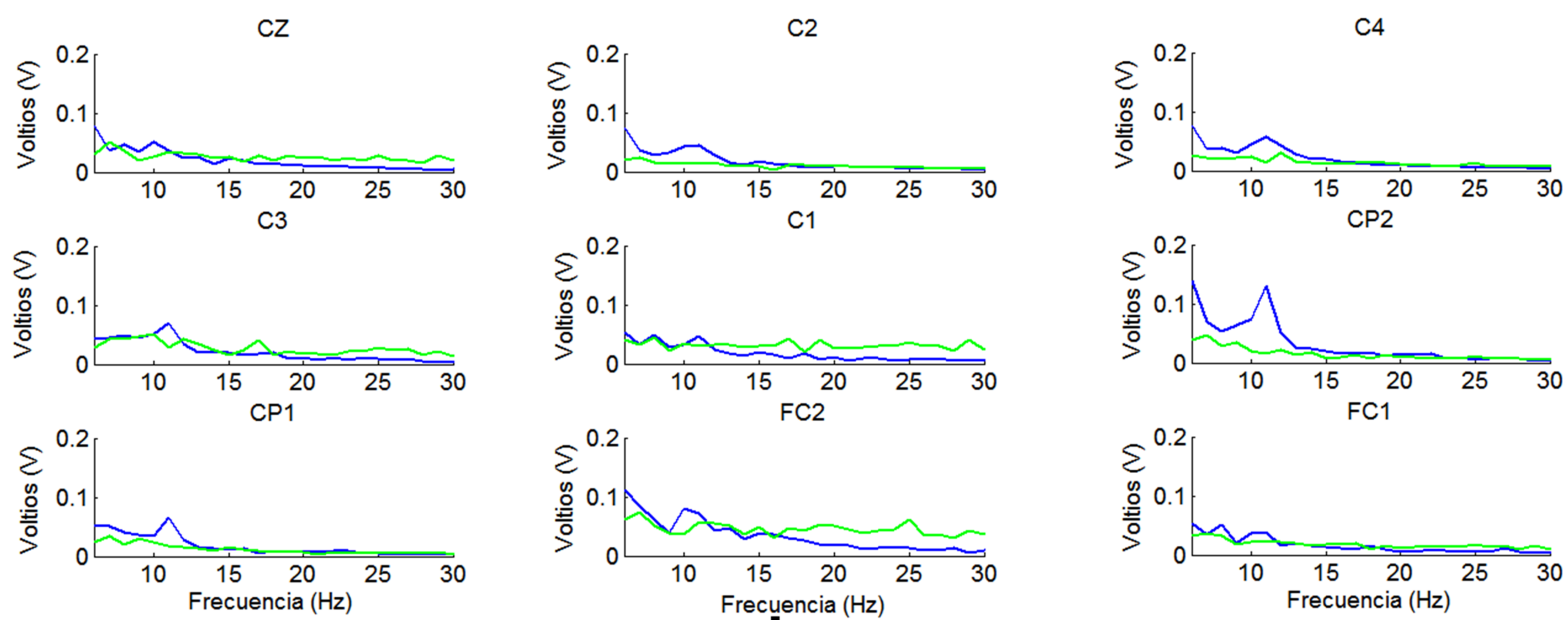

Figura 3: Potencia media espectral de cada electrodo seleccionado durante la imaginación de la marcha para cada escenario: E1 en azul y E2 en verde.
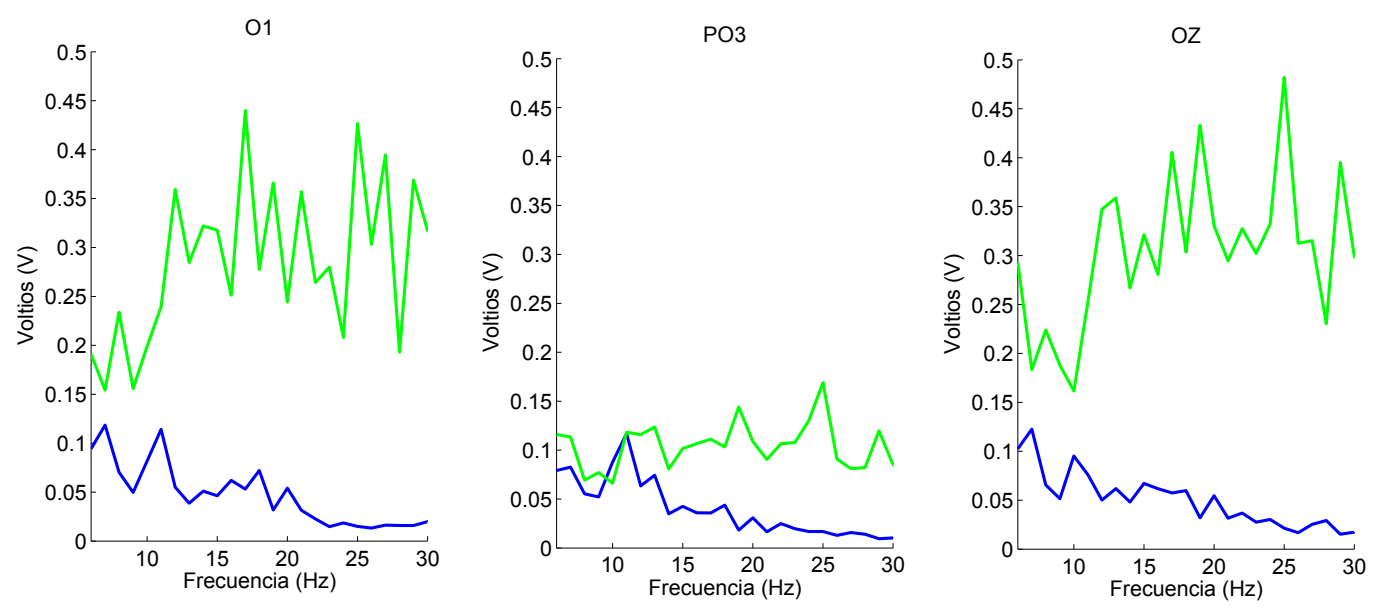

Figura 4: Potencia media espectral de 3 electrodos ruidosos: O1, PO3 y OZ durante la imaginación de la marcha para cada escenario: E1 en azul y E2 en verde.

usuario recibió más o menos luz ésta señal podía haberse alterado; tal y como pasa en un estudio realizado por Smolders et. al. [17]. El electrodo F4, corresponde al área de Brodmann 8.Este área se cree que juega un papel muy importante en los movimientos oculares, por lo tanto, esto explicaría significancia entre las señales [10]. El movimiento de los ojos pudo provocar dichos resultados. El electrodo FC6, pertenece al área de Brodmann 44 y recientemente se ha investigado que dicha área está implicada en la percepción auditiva. Por lo tanto, si en algún momento el usuario escuchó algún ruido, pudo alterar sus señales de EEG.

Por otro lado, varios estudios indican que para que influyera el movimiento del exoesqueleto en las señales de EEG, en E2 se tendría que haber visto cómo la potencia se atenuaba significativamente en los rangos de frecuencia 6-10 Hz y 18-26 Hz [4], [5], [12].

Por lo tanto, se podría concluir que el movimiento del exoesqueleto no influencia significativamente en las señales EEG del usuario y en el futuro, esta tecnología podría utilizarse con personas que han sufrido un ACV. Además, para futuras investigaciones, los electrodos obtenidos como ruidosos se eliminaran a la hora de realizar el filtro Laplaciano para que no genere distorsiones.

\section{Agradecimientos}

Esta investigación ha sido realizada en el marco del proyecto Associate - Decodificación y estimulación de actividad cerebral sensorial y motora para permitir potenciación a largo plazo mediante estimulación Hebbiana y estimulación 
Tabla 1: Significancia t-test.

\begin{tabular}{cc} 
Electrodo & p-value \\
\hline CZ & 0.28 \\
C2 & 0.14 \\
C4 & 0.16 \\
C3 & 0.11 \\
C1 & 0.69 \\
FC1 & 0.21 \\
FC2 & 0.32 \\
CP1 & 0.09 \\
CP2 & 0.12 \\
\hline P7 & 0.25 \\
P4 & 0.12 \\
PZ & 0.14 \\
P3 & 0.18 \\
P8 & 0.26 \\
FP2 & 0.17 \\
FP1 & 0.15 \\
FZ & 0.43 \\
FC5 & 0.10 \\
F3 & 0.21 \\
F4 & $\mathbf{0 . 0 3 *}$ \\
FC6 & $\mathbf{0 . 0 0 *}$ \\
AF3 & 0.14 \\
AF4 & 0.25 \\
CP6 & 0.18 \\
CP5 & 0.17 \\
O1 & $\mathbf{0 . 0 0 *}$ \\
O2 & $\mathbf{0 . 0 0 *}$ \\
OZ & $\mathbf{0 . 0 0 *}$ \\
PO4 & $\mathbf{0 . 0 4} *$ \\
PO3 & $\mathbf{0 . 0 0 *}$ \\
& \\
\hline
\end{tabular}

asociativa pareada durante la rehabilitación de la marcha (con referencia DPI2014-58431-C4-2-R), financiado por el Ministerio de Economía y Competitividad (Plan Estatal de $\mathrm{I}+\mathrm{D}+\mathrm{I}$ ) y por la Unión Europea a través del Fondo Europeo de Desarrollo Regional - FEDER "Una manera de hacer Europa".

Además, el grupo Brain-Machine Interface Systems Lab agradece a la empresa Technaid el préstamo de las instalaciones y el exoesqueleto de miembro inferior H2 para la realización de las pruebas.

\section{English summary}

\section{LOWER LIMB EXOESKELETON INFLUENCE ON EEG SIGNALS}

\author{
Abstract \\ Cerebrovascular accident (CVA) or stroke \\ is one of the most common causes
}

in the world which provokes motor impairment. For this reason, the scientific community is investigating ways to help those affected by stroke. This work uses brain-machine interfaces (BMI) and lower limb exoskeleton technologies to improve the rehabilitation process of CVA patients. In this way, the future patient, will be more involved in his/her rehabilitation therapy. The aim of this work is to study if the generated noise by the exoskeleton affects the EEG signals of the user and therefore, the performance of the BMI can be influenced by it. A power spectrum study of the EEG signals using Fast Fourier Transform was performed. Results show that the movement of the exoskeleton does not produce a significant difference in the power obtained of the selected electrodes. However, a group of electrodes in the occippital area do present significant differences. In the future, we will take this information into account when we use this technology with people that have suffered a CVA.

Keywords: Exoskeleton, EEG, Brain-Machine Interface, noise

\section{Referencias}

[1] Ang, K. K., Guan, C., Chua, K. S. G., Ang, B. T., Kuah, C. W. K., Wang, C., et al. (2011). A large clinical study on the ability of stroke patients to use an EEG-based motor imagery brain-computer interface. Clinical EEG and Neuroscience, 42(4), 253-258.

[2] Ahn, M., Cho, H., Ahn, S., and Jun, S. C. (2013). High theta and low alpha powers may be indicative of BCI-illiteracy in motor imagery. PloS one, 8(11), e80886.

[3] Bortole, M., Venkatakrishnan, A., Zhu, F., Moreno, J. C., Francisco, G. E., Pons, J. L., and Contreras-Vidal, J. L. (2015). The H2 robotic exoskeleton for gait rehabilitation after stroke: early findings from a clinical study. Journal of neuroengineering and rehabilitation, 12(1), 54 .

[4] Cochin, S., Barthelemy, C., Roux, S., and Martineau, J. (1999). 
Observation and execution of movement: similarities demonstrated by quantified electroencephalography. European Journal of Neuroscience, 11(5), 1839-1842.

[5] Crone, N. E., Miglioretti, D. L., Gordon, B., Sieracki, J. M., Wilson, M. T., Uematsu, S., and Lesser, R. P. (1998). Functional mapping of human sensorimotor cortex with electrocorticographic spectral analysis. I. Alpha and beta event-related desynchronization. Brain: a journal of neurology, 121(12), 2271-2299.

[6] Farris, R. J., Quintero, H. A., Murray, S. A., Ha, K. H., Hartigan, C., and Goldfarb, M. (2014). A preliminary assessment of legged mobility provided by a lower limb exoskeleton for persons with paraplegia. IEEE Transactions on neural systems and rehabilitation engineering, 22(3), 482-490.

[7] Field, A. (2013). Discovering statistics using IBM SPSS statistics. sage.

[8] Hanakawa, T., Immisch, I., Toma, K., Dimyan, M. A., Van Gelderen, P., and Hallett, M. (2003). Functional properties of brain areas associated with motor execution and imagery. Journal of neurophysiology, 89(2), 989-1002.

[9] Kollen, B., Kwakkel, G., and Lindeman, E. (2006). Longitudinal robustness of variables predicting independent gait following severe middle cerebral artery stroke: a prospective cohort study. Clinical rehabilitation, 20(3), 262-268.

[10] Künzle, H., and Akert, K. (1977). Efferent connections of cortical, area 8 (frontal eye field) in Macaca fascicularis. A reinvestigation using the autoradiographic technique. Journal of Comparative Neurology, 173(1), 147-163.

[11] McFarland, D. J., McCane, L. M., David, S. V., and Wolpaw, J. R. (1997). Spatial filter selection for EEG-based communication. Electroencephalography and clinical Neurophysiology, 103(3), 386-394.

[12] McFarland, D. J., Miner, L. A., Vaughan, T. M., and Wolpaw, J. R. (2000). Mu and beta rhythm topographies during motor imagery and actual movements. Brain topography, 12(3), 177-186.

[13] Malach, R., Reppas, J. B., Benson, R. R., Kwong, K. K., Jiang, H.,et. al (1995). Object-related activity revealed by functional magnetic resonance imaging in human occipital cortex. Proceedings of the National Academy of Sciences, 92(18), 8135-8139.

[14] Mao, Y., Jin, X., Dutta, G. G., Scholz, J. P., and Agrawal, S. K. (2015). Human movement training with a cable driven ARm EXoskeleton (CAREX). IEEE Transactions on Neural Systems and Rehabilitation Engineering, 23(1), 84-92.

[15] Mukherjee, D., and Patil, C. G. (2011). Epidemiology and the global burden of stroke. World neurosurgery, 76(6), S85-S90.

[16] Shakespeare, T., and Officer, A. (2011). World report on disability. Disabil Rehabil, 33(17-18), 1491.

[17] Smolders, K. C. H. J., De Kort, Y. A. W., and Cluitmans, P. J. M. (2016). Higher light intensity induces modulations in brain activity even during regular daytime working hours. Lighting Research and Technology, 48(4), 433-448.

[18] Rodríguez-Ugarte, M., Iáñez, E., Ortíz, M., and Azorín, J. M. (2017). Personalized offline and pseudo-online BCI models to detect pedaling intent. Frontiers in neuroinformatics, 11, 45 .

[19] Vourvopoulos, A., and i Badia, S. B. (2016). Motor priming in virtual reality can augment motor-imagery training efficacy in restorative brain-computer interaction: a within-subject analysis. Journal of neuroengineering and rehabilitation, 13(1), 69 .

[20] Wodlinger, B., Downey, J. E., Tyler-Kabara, E. C., Schwartz, A. B., Boninger, M. L., and Collinger, J. L. (2014). Ten-dimensional anthropomorphic arm control in a human brain-machine interface: difficulties, solutions, and limitations. Journal of neural engineering, 12(1), 016011.

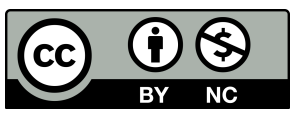
(c) 2018 by the authors. Submitted for possible open access publication under the terms and conditions of the Creative Commons Attribution CC-BY-NC 3.0 license (http://creativecommons.org/licenses/by-nc/3.0/). 